\title{
REFORMAS EDUCATIVAS EN AMÉRICA LATINA: UN ANÁLISIS CRÍTICO
}

\author{
Carolina Guzmán V. \\ Universidad de Valparaíso, Chile
}

\section{DEFINICIONES PRELIMINARES Y TIPOS DE REFORMA}

Primero que todo, se hace necesario definir lo que es una reforma. De acuerdo a Zaccagnini (2004) en educación se habla de reformas cuando se pretende realizar grandes cambios estructurales y/o organizacionales en el sistema educativo, en uno o más de sus niveles.

"cuando se cambia el diseño curricular y los consecuentes planes de estudio; cuando se pretende modernizar al sistema en su conjunto, imprimiéndole una dinámica más ágil y efectiva al funcionamiento institucional, al descentralizar la burocracia central; cuando se pretende elevar la calidad general de la enseñanza, en aras de mejorar el rendimiento académico de los alumnos y disminuir el fracaso escolar; cuando se pretende adecuar y ajustar la formación educativa a las demandas del mercado laboral; cuando se quiere introducir cambios en los estilos pedagógicos de los docentes; cuando se busca transformar las culturas institucionales de las escuelas; cuando se busca mejora) los criterios de organización y gestión institucional de las escuelas, etc." (www.campus-oei.org/revista/ deloslectores/338Zaccagnini.pdf)

En América latina, desde los años 80, se vienen implementando una serie de reformas educativas, con distintos propósitos e impacto. Martinic (2001) hace la distinción entre reformas de primera, segunda y tercera generación que me parece bastante aclaradora. Las primeras, durante la década de los 80 , estarían referidas a la ampliación de cobertura de la enseñanza, y se definen como "reformas hacia fuera", pues hay cambios estructurales en la forma de entregar servicios sociales y educativos desde el gobierno central, de manera que la educación sea administrada y gestionada por las provincias, las comunas o bien por sectores privados. Sin embargo, esta descentralización iría acompañada no sólo por un repliegue del aparato público, sino también por una reducción del gasto en educación. Al haber reducción, aparejadamente se exige cumplir ciertos criterios de eficiencia, especialmente en lo que respecta a la administración de recursos (su mayor y mejor utilización), y su focalización ${ }^{1}$, elementos que continúan estando presentes en la época actual.

La segunda generación de reformas son las de la calidad y la equidad. Muchas veces se dice que el tema de la cobertura está prácticamente superado, aunque siguen siendo los sectores de escasos recursos económicos los que tienen más dificultades para acceder al sistema educativo (Reimers, 2000), especialmente en educación preescolar, secundaria y terciaria. En esta década, la de los 90, se habla de "reformas hacia adentro". Vale decir:

"hacia los modos de gestión y evaluación del sistema; los procesos pedagógicos y contenidos culturales que se transmiten en la escuela... Estas reformas tienen como centro la escuela y la calidad de los aprendizajes. Se promueven políticas que otorgan mayor autonomía y poder a directores y maestros; cambios curriculares y en las prácticas pedagógicas; se diseñan sistemas de incentivos para maestros según desempeño y se realizan mayores inversiones en infraestructura, textos y otros insumos especialmente en las escuelas más pobres de la región. Este nuevo ciclo de reformas está centrado en la calidad de la educación y promueve cambios en

\footnotetext{
${ }^{1}$ Como sabemos, esto sucede también en otros ámbitos de las políticas sociales, de manera de favorecer a las clases menos aventajadas.
} 
el proyecto y gestión educativa de los establecimientos, en la pedagogía, curriculum y sistemas de evaluación" (Martinic, 2001, p. 18).

Finalmente, la tercera generación de reformas que se estaría llevando a cabo en la actualidad estaría centrada aún más en la autonomía de las escuelas y en la denominada descentralización pedagógica, que promueve la transferencia de decisiones pedagógicas y curriculares desde el gobierno central a los centros escolares (García - Huidobro, 1999). Desde esta perspectiva, el foco estaría puesto en el proceso de enseñanza - aprendizaje y de las formas en las que, el propio centro educativo, en estrecha colaboración con los agentes que participan de este proceso, lo pueden optimizar.

Para Martinic (2001), las reformas plantean espacios de interacción entre el nivel central, los niveles intermedios y los centros educativos, a partir de los cuales se plantean visiones frente a las que se puede estar de acuerdo, en desacuerdo, o bien, permanecer indifirente y de ahí que se pueda estar a favor, en contra o al margen de ellas. Algunas de estas visiones son concebidas como válidas por el nivel central y se intenta legitimarlas; pero también existen posturas detractoras que enfatizan otras visiones o bien, voces que llaman a ser cautelosos a la hora de implementar cambios profundos en el sistema educativo e insisten en poner sobre la mesa, las ideologías a la base de la propuesta de transformación.

\section{ANÁLISIS CRÍTICO DE LAS REFORMAS}

No se puede obviar que las reformas surgen en un contexto histórico, social, económico, político, etc., que es necesario tener en cuenta a la hora de diseñar, implementar, evaluar y analizarlas. Para Popkewitz (1994), las reformas educativas implican cuestiones de producción social y de regulación estatal que las van "construyendo". Las reformas, entonces, serían discursos que muchas veces se mantienen ocultos, o implícitos, y que determinan nuestras formas de ver el mundo escolar; visiones que producen valores sociales y relaciones de poder que por tanto no son neutrales; antes, al contrario, dictaminan lo "bueno" de lo "malo" en educación: el buen profesor, el buen estudiante, la buena familia, etc.:

"Los discursos políticamente aceptados organizan la percepción y la experiencia, siendo capaces de crear sistemas de orden dominantes" (Popkewitz, 1994).

Así, estos discursos políticos van configurando y regulando nuestra manera de ver la realidad educativa y se van apoyando en el conocimiento científico, que a su vez les da sustento. En este sentido desde la investigación se dice lo que es correcto, apropiado, etc., y es un discurso que por ser científico posee la investidura de válido.

Pero además, estos discursos regulan nuestras prácticas cotidianas en la escuela (a través de las normas, las reglas, la evaluación; en definitiva, la normalización), sin ser conscientes de ellos la mayoría de las veces:

"Las palabras son parte de un sistema de reglas de la escuela con el que se condiciona qué tipo de discurso educativo es posible, quiénes pueden considerarse interlocutores serios y autorizados, y cómo han de construirse el deseo, las aspiraciones y la cognición. Además, las palabras emblema de la reforma se sitúan dentro de las estrategias que determinan qué preguntas son las que deben hacerse: definiendo cuáles son los fenómenos de la práctica y configurando cómo debe gestionarse y ordenarse la experiencia en tanto que objetos para la indagación, esto es, dónde hay que mirar y cómo esa mirada ha de concebir las cosas del mundo" (op. cit)

Llegados a este punto, considerando que los dos ejes centrales de las actuales reformas educativas son calidad y equidad educativa, las que a su vez requieren de la autonomía del centro escolar, quisiera 
detenerme y profundizar en estos conceptos para intentar desvelar ciertas ideologías de base, que muchas veces permanecen ocultas.

\section{$2.1 \quad$ Educación de Calidad}

Las reformas educativas son valoradas a partir de un espacio social que es el entorno sociocultural, económico y político en el cual se inserta el proceso educativo. En este sentido, la calidad educativa es una construcción social, históricamente determinada, cuya concepción obedece a condiciones económicas, políticas, culturales y sociales determinadas; de ahí que existan visiones que puedan ser hasta antagónicas a la hora de valorarlas.

Ahora bien, cuando hablamos de reformas educativas y de calidad de la educación, tal como señalan Gimeno (1992) y Bolívar y Rodríguez (2002), resulta difícil no estar de acuerdo frente a este ideal imaginario de progreso. Sin embargo, desde una pedagogía más crítica, se nos llama a ser cautelosos, a realizar un análisis de estas retóricas de la reforma y a estar más atentos a los discursos ideológicos de base; de ahí que se vuelva necesario saber desde qué enfoque se la está examinando.

Desde una perspectiva eficientista, Rodríguez (2003) plantea que la calidad educativa es aquella "manera de administrar correctamente los escasos recursos asignados a las instituciones de enseñanza a efectos de alcanzar el máximo de resultados educativos posibles". Lo anterior necesariamente implica el aumento de control sobre el funcionamiento del sistema, administrar correctamente los insumos y ser eficiente en la asignación de los recursos. Se entiende así la calidad como rentabilización y rendición de cuentas para optimizar esfuerzos, tiempo y recursos - lo que también se conoce como "accountability" (Gimeno, 1992).

A partir de otro enfoque podríamos analizar la calidad educativa como eficacia, en términos del valor añadido, definido como la "medida del incremento del rendimiento de un estudiante, producida por el efecto de la escuela, una vez eliminada la influencia de las características de entrada (background) del mismo" (Fernández y González, 1997). Algunos modelos más cuantitativos y lineales en educación han intentado desde esta postura, aislar ciertas variables del proceso educativo para explicar causalmente un buen o mal rendimiento. Por ejemplo, se habla de profesor efectivo (Arancibia, 1992) o de escuela efectiva, refiriéndose a aquellos que poseen ciertas características consideradas claves para alcanzar mejores resultados de aprendizaje. Popkewitz (1994) señala en todo caso que esto es cuestionable, pues, una vez identificados los rasgos "positivos o deseables" no es posible "exportar sus cualidades a otras escuelas como si se tratara de trasladar bienes físicos").

Existen otros enfoques, más bien centrados en los procesos internos del sistema educativo y los productos de éste a partir de elementos cualitativos del proceso mismo y de los resultados de aprendizaje. Es así como Coombs (en Rodríguez, 2003) se centra en el proceso de enseñanza - aprendizaje, afirmando que:

"la calidad tiene que ver con la coherencia de lo que se enseña y se aprende, con el grado de adecuación a las necesidades de aprendizaje, presentes y futuras, de los aprendices concretos, habida cuenta de sus circunstancias y expectativas particulares. La calidad de la educación exige contemplar, además, las características de los elementos que integran el sistema educativo: estudiantes, instalaciones, equipamiento y otros medios, sus objetivos, contenidos de la programación y tecnologías educativas; también los entornos socioeconómicos, culturales y políticos". 
Elliot (en Rodríguez, 2003), por su parte, esgrime una postura que podemos llamar de "coherencia valórica" y que yo, por cierto, comparto; para este autor, la calidad educativa va a depender del grado de concordancia que exista con los valores educativos como finalidad misma del proceso educacional; pudiéramos decir que para este autor, la calidad guarda relación con el proceso formativo propiamente tal:

"estos valores no son valores instrumentales, como la efectividad y la eficiencia, sino conceptualizaciones de los potenciales humanos que un proceso educativo ha de aspirar a fomentar y desarrollar en los alumnos; por ejemplo, potenciales para comprender el significado de ciertos tipos de acontecimientos y situaciones; para la formación de un pensamiento crítico, reflexivo e imaginativo; para la apreciación de los valores humanos; para una acción inteligente y sensata en situaciones humanas complejas e impredecibles'.

Como vemos, la calidad es un concepto polisémico y depende del ángulo desde el que se le enfoque para definirla y medirla. Según la manera de concebirla serán generados distintas formas de evaluarlas: algunas rescatarán una visión más cuantitativa (por ejemplo el enfoque eficientista o de costo resultado) y otras una visión más cualitativa centradas en los procesos internos y de optimización y transformación del proceso educativo.

Ahora bien, si nos centramos en un análisis un tanto más crítico de la calidad educativa propuesta por las reformas educativas actuales en América latina, nos encontramos con autores que señalan que ésta, más que nada, se reduce a la obtención resultados duros (rendimiento) acompañada por una visión eficientista, de administración de recursos (el hacer más con menos). Así, la escuela y sus docentes deben lograr que los estudiantes desarrollen competencias y que obtengan un rendimiento altamente competitivo, que obviamente sólo es evaluado en términos cuantitativos (pruebas de rendimiento estándares). Y esto a pesar de que el "discurso divulgado" promueva prácticas pedagógicas constructivistas que concentran su atención en el proceso de aprendizaje del alumno/a. Pues bien, lo que prevalece son los resultados obtenidos, los índices de eficiencia y no las formas en que se va "construyendo el aprendizaje".

El tema de la competitividad, según Zaccagnini es una estrategia que permite poner a la educación a la altura del proceso de mundialización: en la medida en que los alumnos desarrollan ciertas competencias, son funcionales al mercado laboral y productivo, que también es cada vez más competitivo. Desde aquí se vuelven tan usuales y "normales" los rankings de escuelas: desde la mejor a la peor y frente a las cuales, los consumidores "deciden", cuál es más apropiada para ellos, según los estándares de calidad - competitividad. Mi pregunta es ¿hay elección posible para el caso de los más pobres?; o bien, ¿puede decirse que todas las escuelas están en igualdad de condiciones para ofrecer un servicio que aparenta ser similar (en términos de objetivos de aprendizaje, prácticas pedagógicas o duración de la jornada escolar) pero que en realidad no lo es? Pues las diferencias (en términos de rendimiento, salario de los docentes, equipamiento, infraestructura, número de alumnos, etc.), saltan a la vista y la realidad es que mucha capacidad de elección y decisión, para el caso de los sectores más desfavorecidos, no existe; de hecho, lo más probable, es que las familias escojan aquellas escuelas que se aproximen geográficamente a su lugar de residencia, en un sector pobre claro está.

Además, desde una visión eficientista el tema de los recursos financieros se vuelve preponderante y por tanto la reforma en su trasfondo, conlleva más bien una política de corte economicista: no debemos olvidar que en muchos países de América Latina, las reformas se realizan al mismo tiempo que se llevan a

\footnotetext{
${ }^{2}$ El constructivismo enfatiza el carácter social, activo y comunicativo de los sujetos implicados en la construcción de significados, conocimientos y funciones psicológicas superiores. Esto ocurre al interior del salón de clases, en una situación interactiva y con una intervención deliberada por parte de un adulto experto, es decir, el docente (Coll, 1990).
} 
cabo políticas de ajuste económico, y por ende, quedan supeditadas generalmente a una reducción presupuestaria. Por tanto, "todo parecería solucionarse con un mejor "management". Se trasladan enfoques de la realidad empresarial a las escuelas" (Martinic, 2001, p. 30).

Por otro lado, el llamado a la calidad educativa de las reformas ha conllevado una necesidad considerada como imperativo: la profesionalización docente, que revisaré a continuación y frente a lo cual, como ya se ha podido vislumbrar, hay que ser cautos.

\subsubsection{Calidad y profesionalización docente}

Para Sarramano, Noguera y Vera (1998) lo que distingue a los profesionales de la educación son ciertos conocimientos y competencias que lo habilitan para desarrollar unas funciones y tomar decisiones pedagógicas en pos de la tan mentada calidad educativa. Es así como tres serían las funciones pedagógicas: de docencia, de apoyo al sistema educativo y de investigación (el énfasis estaría puesto en la primera de ellas). Para ejercer estas funciones de manera autónoma, el docente requiere de cierto conocimiento y técnicas de actuación, para lo cual debe ser preparado.

Como vemos, se evidencia una relación clara entre el docente, su actuación y preparación y la calidad educativa; es así como uno de los temas recurrentes en la literatura pedagógica actual, es el de la profesionalización del profesor, tema que se considera íntimamente vinculado a la mejora de la calidad de la educación pretendida en la mayoría de las reformas educativas actuales en la región.

Pero aquí cabe preguntarse ¿a qué intereses o condiciones económicas, sociales, políticas, históricas etc., puede responder este llamado a la profesionalización docente?

Según Martínez (1994), nos percatamos que esta retórica institucional acerca de la calidad, el rendimiento, la competitividad, etc, enmascara nuevas y crecientes demandas al profesorado que se traducen en una creciente descualificación y proletarización del trabajo docente a través de la estandarización de competencias y habilidades de enseñanza y la división social del trabajo entre quienes piensan y quienes ejecutan. Otros autores, como Ortega (1990), concuerdan con esta visión y señalan que el profesionalismo es una reducción del docente al manejo de procedimientos técnico - instrumentales; o bien, Popkewitz (1990), que señala que precisamente la formación del profesorado se ha circunscrito a adquisición de contenidos y al manejo de destrezas fragmentadas, generando así un menoscabo intelectual. Desde su perspectiva, el resultado histórico de las reformas ha sido la limitación de las atribuciones y competencias docentes: las consideraciones éticas e intelectuales han sido sustituidas por las destrezas de administración (gobierno de aula, disciplina). Así, las llamadas reformas para la calidad de la educación han recargado el trabajo de los profesores y aumentado el control de las prácticas docentes (rendición de cuentas): en la medida que los profesores son los responsables de los resultados de aprendizaje, es necesario controlarlos, medir constantemente.

Para Zamora (2003), las reformas en la calidad de la educación dan un mayor peso a la Administración y los directores en el diseño de la práctica educativa, en desmedro del profesorado que pierde peso en la toma de decisiones, de posicionamientos y de transformaciones políticas:

"Los docentes pasan pues a ser más ejecutores de las tareas docentes, ahora con el viejo barniz de técnicas y quedan excluidos de la participación más independiente en la política educativa más general". 
Desde esta postura, es necesario formar al profesorado, o perfeccionarlo, para suplir supuestas carencias que impiden alcanzar una educación de calidad. Pero eso no es todo, además, la política de profesionalización se basa en la obligación del docente de acreditar permanentemente sus competencias profesionales, las que debe desarrollar a partir de la capacitación que ofrece el nivel central de acuerdo al perfil del "buen profesor" - que es aquel que obtiene adecuados resultados de aprendizaje evaluados a través de pruebas estándares -, pero que no da lugar a que el docente tome sus propias decisiones, sino más bien, son otros (los expertos), lo que deciden lo que está bien y cómo se debe hacer. Según Zaccagnini (2004), se impone un modelo de corte meritocrático, que:

"subordina al docente a la aprobación compulsiva de saberes y conocimientos prescritos por los "expertos", descontextualizados de la realidad social y cultural del hecho educativo".

Como vemos, en ningún caso, desde esta perspectiva, puede considerarse al docente como un profesional autónomo que toma decisiones pedagógicas. Así, pedirle al profesor que colabore en la reforma para lograr la calidad educativa resulta contradictorio cuando en realidad lo que se les pide es que se comprometan con una visión que los concibe como sujetos pasivos, sumisos, obedientes: buenos técnicos que aplican lo que proponen los expertos.

\subsection{Educación de calidad para todos: los principios de equidad y descentralización}

A pesar del llamado a la calidad y a la equidad educativa, es un hecho que persiste la desigualdad tanto en el acceso como en la calidad de los resultados de aprendizaje (Martinic, 2001).

De acuerdo con la literatura, sobre el tema podemos encontrar dos posturas en torno a la relación existente entre educación y desigualdad social. Una postura que considera a la educación como "reproductora" de las desigualdades sociales (Cox, 1984; Tedesco, 1985) y una postura más optimista que considera la educación como facilitadora en el acceso a mejores condiciones de vida, o a lo que se ha llamado "movilidad social" (Weinstein, 1998). Más aún, algunos plantean la educación como "la" variable que discrimina en los procesos de inclusión/exclusión sociales (Dávila, 1998).

Según la primera postura, la educación no necesariamente permite acceder intergeneracionalmente a una mejor calidad de vida, especialmente en los sectores más desfavorecidos social y económicamente, siendo cinco los procesos educativos que transmiten la desigualdad (Reimers, 2000):

- El acceso diferencial a distintos niveles educativos para los pobres v/s los no pobres: si bien la mayoría accede a la educación primaria, sólo algunos la terminan y continúan en el nivel secundario y terciario, siendo estos últimos los que provienen de sectores con mayores ingresos.

- El tratamiento diferencial que se da en las escuelas a los distintos estudiantes, que da una mayor ventaja a los estudiantes procedentes de sectores aventajados económica y socialmente: cuentan con profesores más capacitados que dedican más tiempo a tareas de enseñanza, "la organización de la escuela está más centrada en apoyar el aprendizaje de los alumnos y hay más recursos para facilitar la tarea de los maestros" (p. 25). Precisamente, según este autor, este aspecto se relaciona con el mencionado anteriormente pues las "diferencias cualitativas entre contextos de aprendizaje explican la variación en los resultados 
de aprendizaje entre escuelas, y que los estudiantes de menores ingresos tengan menor probabilidad de culminarla educación primaria en el tiempo previsto" (p. 39) ${ }^{3}$.

- La segregación social que se da en las escuelas: los estudiantes conviven y aprenden a relacionarse con estudiantes de un nivel sociocultural similar y de acuerdo al autor, "esto dificulta a los hijos de hogares de menores ingresos adquirir un capital social en forma de relaciones con personas con mayor capital cultural'(p. 25)

- La no existencia de un proyecto educativo que trate directamente la desigualdad como un problema que debe resolverse.

- Finalmente, los esfuerzos que invierten los padres de nivel socioeconómico aventajado: en primer lugar destinan mayor cantidad de recursos económicos para que las actividades que desarrollan sus hijos sean óptimas; en segundo lugar, y de acuerdo al autor, estos padres sostienen mayor cantidad de conversaciones con sus hijos que estimulan un tipo especial de pensamiento que los impulsa a acceder a más oportunidades ${ }^{4}$.

A través de estos procesos o mecanismos podemos observar claramente cómo se reproduce o mantiene la desigualdad social. También es importante señalar que a la base de ellos existen creencias, valoraciones, ideologías conscientes o inconscientes que dan soporte a la mantención de la desigualdad. Pérez (2000) por ejemplo, alude a las concepciones y actitudes que tienen algunos profesores con respecto a la "falta" de habilidad de ciertos estudiantes, falta de habilidad académica o conductual que se debería más a factores personales que escolares y que llevaría a "marcar la diferencia" entre los alumnos, a su jerarquerización en torno al rendimiento o el comportamiento. Otra actitud que muchas veces prevalece en el profesorado, es una especie de descalificación hacia las prácticas familiares de los niños y jóvenes de sectores desfavorecidos que serían deficitarias, y a sus concepciones en torno a la educación, considerándolas como opuestas a las de la escuela (por ejemplo disciplina escolar v/s permisividad en el hogar, o bien centración en el proceso de enseñanza-aprendizaje, considerado como global y complejo, v/s centración en los resultados escolares) De acuerdo a esta postura los padres debieran delegar completamente la educación de sus hijos en los profesores, sin cuestionarla o fiscalizarla.

Finalmente, otra visión que de acuerdo a la autora, promueve el rol de la escuela en la mantención de las desigualdades sociales, es aquella que identifica las condiciones socioculturales deficitarias como determinantes sociales que impiden la promoción y movilidad social de los alumnos; en este contexto, la escuela y la labor pedagógica del profesorado, no tendría muchas posibilidades de revertir la situación. Este planteamiento estructural no "cree" en modelos pedagógicos que promuevan una enseñanza más igualitaria y democrática, "pues la naturaleza reproductora de la escuela es asumida como prácticamente inevitable" (p. 204)

Frente a la postura antes expuesta, encontramos una visión más bien optimista, que plantea que la educación es una fuente de mayor equidad social, un vector fundamental para el desarrollo equitativo y la competitividad de los países. Es así como:

\footnotetext{
${ }^{3}$ Algunas consecuencias directas de este hecho, para los estudiantes de bajos ingresos según este autor, son: menores competencias académicas para continuar sus estudios secundarios lo que puede traer como consecuencia la repitencia o abandono escolar.

${ }^{4}$ En este sentido, el autor habla de recursos o capitales económicos y culturales.
} 
"[La educación] es el único instrumento que realmente crea una base sólida a las personas para lograr su propio desarrollo, su propia inserción y su propia solución al problema de la calidad de vida. Todas las otras van a ser un poco asistenciales (...) Pero si hay una acción que iguala a los individuos, es la educación" (Fernández, 1996; p.126).

Estos planteamientos han sido sostenidos y avalados por los discursos internacionales como nacionales y es precisamente esta postura la que esgrimen los diversos gobiernos, especialmente los menos desarrollados, cuando impulsan reformas educativas en los años 90. Las estrategias impulsadas por el gobierno central en este sentido guardan relación con (Rivero, 2000):

- Políticas compensatorias intersectoriales.

- Generación de programas de naturaleza compensatoria ${ }^{5}$.

- Implementación de estrategias de discriminación positiva, es decir, a favor de núcleos poblacionales en situación de riesgo. Aquí se trata de destinar más recursos y de organizar proyectos y programas explícitamente destinados a favorecer la educación de estratos en situación de pobreza; con ella se intenta "conseguir una educación diferenciada para obtener resultados semejantes"(p. 130).

- Un aumento considerable de los recursos asignados a educación, desde fuentes. nacionales (públicas y privadas) y préstamos internacionales.

- Búsqueda de acuerdos nacionales para asumir la educación como política de Estado.

- Cambios a nivel de gestión, favoreciendo la descentralización administrativa y pedagógica. Es decir, se pretende otorgar mayores atribuciones a los Centros Educativos desconcentrando el poder y toma de decisiones y disminuyendo la escala burocrática central.

Ahora bien, como podemos observar, la misma idea de eficiencia de recursos aparece cuando analizamos el tema de la equidad: se habla de discriminación positiva y focalización en los sectores de más escasos recursos, mecanismo que puede comprenderse perfectamente en un contexto político que necesita reducir el gasto fiscal debido a las importantes deudas de los países (me refiero a América Latina)

Para Reimers (2000) lograr la igualdad de oportunidades educativas es un acto político pues implica generar proyectos que mejoren las sociedades latinoamericanas para hacerlas más justas y democráticas de manera que todos puedan tener garantizados sus derechos humanos y vivir en comunidad. En este gran proyecto, debe estar implicado el gobierno, instituciones públicas y privadas, profesores y familias, la comunidad en general, dejando a un lado actitudes paternalistas y autoritarias que han prevalecido en la base de las políticas compensatorias y que mantienen el status quo de la estratificación social y económica. Mas yo me pregunto, ¿realmente los grupos privilegiados están convencidos de la de la igualdad fundamental de todos los seres humanos y de su derecho a recibir una educación de calidad? ¿Se promueven decisiones políticas que se traduzcan efectivamente en una mayor equidad educativa, o sólo queda como un ideal utópico, en una sociedad caracterizada por una gran desigualdad y que sólo pretende equiparar a los individuos en sus competencias para que sean más productivos y mejoren la competitividad económica?

\footnotetext{
${ }^{5}$ Se trata de focalizar la inversión en poblaciones pobres, atendiendo aquellas variables que han demostrado estar vinculadas con la calidad de los aprendizajes básicos. Hay dos visiones de fondo respecto a la compensación para lograr la equidad educativa: una que organiza y ejecuta estos programas en función del déficit cultural., y la otra que se fundamenta en el criterio de la diversidad cultural. (Rivero, 2000).
} 
En cuanto a la descentralización en sus aspectos administrativos y pedagógicos ${ }^{6}$, queda la sensación de que, debido a que el gobierno no puede hacerse responsable de la educación en sus aspectos económicos y sociales (repliegue del estado que obedece a un modelo neoliberal) y de los resultados de aprendizaje, promueve la autonomía de las escuelas y la toma de decisiones educativas de la comunidad y de las familias. Pero es una autonomía bastante parcial, pues se insta a los municipios y a las escuelas a administrar la pobreza de los recursos y mejorar el rendimiento; se llama a que tome decisiones pedagógicas, pero, a la hora de evaluar y capacitar a los profesores, los lineamientos provienen del nivel central. La autonomía se reduce en este caso a dejar toda la responsabilidad de la calidad educativa a una escuela, y el estado se desentiende. Los principales perdedores en este caso, son las escuelas que históricamente cuentan con menos recursos y los profesores, quienes se transforman en "la causa" de una mala educación: si hay malos resultados de aprendizaje es porque las escuelas y los profesores no han sabido aplicar de manera correcta aquello que se les ha prescrito.

En realidad, según Zaccagnini, la tan mentada descentralización en sus aspectos administrativos y económicos, conlleva nociones de mercado y de elección y:

"representa la tendencia del Estado a reducir paulatinamente su responsabilidad básica de garantizar económicamente los servicios educativos. Los alumnos pasan a ser categorizados como clientes, quienes de la manos de sus padres elegirán aquellas escuelas cuya oferta se ajuste mejor a sus necesidades e intereses, por ello se introduce el concepto de "competitividad» como regulador dinámico de la oferta educativa... Como corolario, es obvio que se necesita una nueva función del docente para este modelo escolar empresarial... En suma, lo que se desprende fundamentalmente de este análisis es el replanteo de la distribución de las responsabilidades públicas (en este caso la política educativa) que le caben al Estado y a la sociedad civil" (p. 9).

\section{ALGUNAS REFLEXIONES FINALES}

Martinic (2001) propone una serie de iniciativas para mejorar los procesos de reforma; entre ellas, mejorar los canales mediacionales y de comunicación entre los que "piensan" las reformas (nivel central) y los que la ejecutan, de manera de lograr un discurso de consenso frente a ella. $O$ bien mejorar los procesos de gestión y organización tanto a partir de las coordinaciones entre el nivel central con el local, como en las instituciones educativas mismas para desburocratizarlas. Se confiere importancia a la optimización de los procesos de gestión, de participación de todos los actores, etc.

Considero relevantes estas propuestas, pero creo que obedecen a una visión un tanto "ingenua" de la realidad. Es así como mi preocupación se centra en las dificultades que tenemos para hacer un análisis crítico de la realidad educativa y de las reformas: muchas veces no tenemos en cuenta que todas las políticas gubernamentales incluidas las educativas, se insertan en un contexto político, económico, social e histórico determinado, que no puede ser "puesto entre paréntesis", a la hora de diseñar, implementar y evaluar el impacto de las reformas. En este sentido, es un hecho que un modelo económico como el neoliberal, está muy presente en la actualidad, por lo que conceptos como calidad, equidad y descentralización quedan reducidos a eficiencia, competitividad, repliegue del estado y regulación del mercado. Puede ser que la prevalencia de este tipo de discursos - que es precisamente el manejado por los grupos de poder económico - , finalmente sea una de las causas relevantes por las que las reformas educativas y otras tantas, fracasan. No olvidemos, como dice Popkewitz, que los discursos, crean y regulan nuestra realidad; en este sentido, muchas veces son discursos de poder hegemónicos. A mi parecer, no

\footnotetext{
${ }^{6}$ Para García - Huidobro (2000), la descentralización pedagógica promueve la transferencia creciente de responsabilidad y autonomía
} 
está demás preguntarnos, ir más allá, lograr una nueva aproximación o "sospechar" (como diría Foucault) de las reformas educativas e intentar descifrar las concepciones e ideologías de base, que las sustentan y que dan sentido a los cambios educativos propuestos; desde aquí, a mi entender podremos trasformar y mejorar la realidad educativa.

\section{BIBLIOGRAFÍA}

ARAnciBia, V. Efectividad escolar, un análisis comparado. Estudios Públicos, 1992, 101-125.

BoLívar, A. y RodRíGUEZ, J. Reformas y retóricas. La reforma educativa de la LOGSE. Málaga: Aljibe, 2002

CoLL, C. Aprendizaje escolar y construcción del conocimiento. Barcelona: Paidós, 1990

Cox, C. Clases, reproducción cultural y transmisión escolar: una introducción a contribuciones de Bourdieu y Bernstein. CIDE: Santiago de Chile, 1984.

DÁvILA O. Exclusión social y juventud popular. Ultima década, (8), 1998 89-108

FERNÁNDEZ, G. Superación de la pobreza y educación: Una mirada desde lo local. Ultima década, (5), 1996, 105-136.

FERnÁNDEZ M. y GonZÁLEZ, A. Desarrollo y situación actual de los estudios de eficacia escolar. Revista Electrónica de Investigación y Evaluación Educativa, 3, (13), 1-20, 1997. Consultado en [http://www.uv.es/RELIEVE/v3n1/RELIEVEv3n1_3.htm], Mayo de 2004

García-Huidobro, J.E. (documento no publicado). La Escuela y la calidad de la educación. Santiago de Chile, 2000

García-Huidobro, J.E. y Cox, C.. La Reforma Educacional Chilena: 1990-1998. Visión de conjunto. En J. E. García-Huidobro (editor) La Reforma Educacional Chilena., 7-46. Madrid: Popular, 1999

GIMENO, J. Reformas Educativas. Utopías, retórica y práctica. Cuadernos de Pedagogía, (209), 1992, 162167.

MARTínEZ, J. Los colectivos críticos de profesores y profesoras en el Estado Español. Investigación en la Escuela, (22), 1994, 7-22

MARTINIC, S. Conflictos políticos e interacciones comunicativas en las reformas educativas en América Latina. Revista Iberoamericana de Educación, (27), 2001, 17-33.

Ortega, F. La indefinición de la profesión docente. Cuadernos de Pedagogía, 186, 1990, 67-70

Pérez, C. La escuela frente a las desigualdades sociales. Apuntes sociológicos sobre el pensamiento docente. Revista Iberoamericana de educación, (23), 2000, 189-212.

POPKEWITZ, T. Profesionalización y formación del profesorado. Cuadernos de pedagogía, (184), 1990, 105110

PoPkEWITZ, T. Política, conocimiento y poder: algunas cuestiones para el estudio de las reformas educativas. Revista de educación, (3005), 1994. Consultado en 
[www.reduc.cl/raes.nsf/4211b585503d5ece04256843007c08e2/e762aa3e3e13b0bb04256a4900580 544/\$FILE/7542.pdf ], Mayo de 2004

ReIMERS, F. Educación, desigualdad y opciones de política en América Latina en el siglo XXI. Revista Iberoamericana de educación, (23), 2000, 21-50.

RIVERO, J. Reforma y desigualdad educativa en América Latina. Revista Iberoamericana de educación, (23), 2000, 103-133.

RODRíGUEZ, E. Reforma de la educación superior en América Latina. Algunas reflexiones sobre calidad de la enseñanza universitaria, formación del profesorado y las reformas institucionales actuales a partir del análisis del debate en la universidad uruguaya, 2003 Consultado en [http://www.cica.es/aliens/revfuentes/mono_03.htm], Mayo de 2004.

SarRamona, J., Noguera, J. y Vera, J. ¿Qué es ser profesional docente? Revista de Teoría de la Educación, 1998. Consultado en [http://www.segciencias.com.ar/profesional.htm], Mayo de 2004

TEDEsco, J. Reproductivismo educativo y sectores populares en América Latina. Educação na América Latina: Os Modelos Teóricos e Realidade Social, São Paulo: Cortez, 1985

WeINSTEIN, J. La búsqueda del tesoro perdido. Educación y Juventud en América Latina. Proyecto Principal de Educación, 45, 1998, 71-87.

ZACCAGINI, M. Reformas educativas: espejismos de innovación. Revista Iberoamericana de Educación (s/a). Consultado en [www.campus-oei.org/revista/ deloslectores/338Zaccagnini.pdf], Mayo de 2004

ZamorA, B. Andando no siempre se hace el camino. Las paradojas entre la formación y el rol del profesorado en la legislación educativa en España. $X$ Conferencia de Sociología de la Educación. Valencia, Septiembre 2003. Consultado en [www.campus-oei.org/revista/ deloslectores/338Zaccagnini.pdf], Mayo de 2004. 


\title{
Contactar
}

Revista lberoamericana de Educación

\author{
Principal OEI
}

